\title{
Editorial
}

Skin

Appendage

Disorders
Skin Appendage Disord 2019;5:197-200

DOI: 10.1159/000497044
Received: December 21, 2018

Accepted: January 16, 2018

Published online: March 1, 2019

\section{Innovative Physical Approaches for Onychomycosis: Peeling, Lasers and Beyond}

\author{
Sidharth Sonthalia ${ }^{a}$ Mohamad Goldust ${ }^{b}$ \\ ${ }^{a}$ Department of Dermatology and Dermatosurgery, Skinnocence: The Skin Clinic and Research Center, Gurugram, \\ India; ${ }^{b}$ Mazandaran University of Medical Sciences, Sari, Iran
}

Cutaneous mycotic infections are becoming increasingly difficult to manage owing to a plethora of factors, an important one being the gradual but steadily increasing development of resistance in the causative fungal species against multiple standard antifungal drugs. Onychomycosis (ONM) presents a peculiar situation where despite retained drug susceptibility by the causative fungus, the effect of antifungal drugs is suboptimal. Although genetically determined anergy, or lack of immune response to specific fungal antigens, have been reported in patients with chronic dermatophytosis (e.g., ONM is often ignored by the affected patients for years before seeking treatment), factors such as pathogen virulence, drug resistance, and most importantly formation of biofilms by Trichophyton rubrum and Candida spp. contribute to the suboptimal or nonresponse to drugs in ONM [1]. Microbial pathogens including fungi have become smarter and more civilized by secreting a thick layer of extracellular polymeric substance composed of polysaccharides, proteins, lipids, and extracellular DNA, which constitute the biofilm within which they thrive and survive, supporting each other in a colonial fashion. In addition to the formation of a robust impenetrable mechanical barrier that insulates their colonies from

\section{KARGER}

() 2019 S. Karger AG, Basel

E-Mail karger@karger.com

www.karger.com/sad the environment (especially topical antifungals), biofilms provide increased communication, virulence, better metabolic cooperation, and concerted gene expression, leading to increased resistance to antifungal agents (for example, by activation of drug efflux pump genes). Moreover, the host immune system is unable to access the fungi encased in a mature biofilm, leading to tissue damage from an ineffectual immune response with persistence of infection [1, $2]$. Therefore, disruption of fungal biofilms before or concomitant with antifungal drug therapy is essential for successful eradication of infection. The biofilm can be tackled at three steps of its formation and maturation. Fungal biofilms have a complex three-dimensional structure and diverse spatial heterogeneity. Typically, biofilms are embedded in an extracellular matrix encased within exopolymeric material with a microcolony/water channel architecture.

\section{The "Fungal Biofilm Life Cycle"}

The life cycle of a fungal biofilm formation consists of initial attachment (adherence and initiation), proliferation, maturation, and ultimately dispersion [3]. The first 
step is the adherence of yeast form cells to a substrate like the nail. This is followed by proliferation of the yeast cells into filamentous forms (hyphae and pseudo-hyphae) and their growth over the surface. Accumulation of extracellular matrix around nested fungal colonies results in maturation of the biofilm with a high biomass composition.

\section{Fungal Biofilm Inhibitors}

Many compounds synergize with conventional antifungals to enhance the overall fungal killing. While some have shown intrinsic antibiofilm activity (type I potentiators) like Farsenol, auranofin, gentian violet, hexachlorophene, biothionate disodium, nitroxoline, toremifene, miltefosine, and silver nanoparticles, others enhance the antifungal activity of drugs like azoles (type II potentiators) without having any robust antibiofilm activity of their own such as calcineurin inhibitors, amantadine derivatives, 1,3-benzothiazoles, and artesunate amongst others [3]. Apart from these, a plethora of naturally derived molecules have displayed antibiofilm activity against Candida spp. at different stages of the biofilm life cycle including different plant alkaloids, phenolic compounds, xanthochymol, garcinol, propolis, curcumin, and essential oils (menthol, cinnamon) [3-14].

The basic approaches to disrupt a microbial biofilm, whether bacterial or fungal, are similar. They include mechanical removal (surgical debridement), chemical disruption of the extracellular polymeric substance components (echinocandins, DNase I enzyme, terpinen-4-olloaded lipid nanoparticles, povidone-iodine), thermal disruption (selective photothermolytic effect achieved by laser devices), photochemical damage induced by generation of reactive oxygen species and singlet oxygen using antimicrobial photodynamic therapy, or low-frequency surface acoustic waves. Although the majority of the approaches have shown in vitro and/or in vivo success against bacterial and yeast biofilm disruption, the same may be modulated and fine-tuned to cover dermatophytes [1].

The lack/poor grade of evidence of most of the approaches mentioned above (excepting lasers) for damaging biofilms of dermatophytic ONM - and the cost of laser treatment - constitute huge impediments in using them to treat drug-recalcitrant ONM caused by T. rubrum. The concept of antifungal nail peeling with concomitant regular use of topical ciclopirox olamine/amorolfine lacquer is evolving as a cost-effective alternative, not just as an adjunctive therapy with oral antifungals but particularly for ONM in special populations such as the elderly, diabetics, and generally debilitated patients who are poor candidates for oral therapy. Nail peeling with glycolic acid, conceptually introduced for improving surface abnormalities of nails by Banga and Patel [15], was followed by another trial that compared glycolic acid with low-concentration croton-oil-free phenol combination peel for trachyonychia [16]. However, the role and use of nail peeling specifically for ONM has not been reported to date. In the section on Novel Insights in this issue of the journal, the successful eradication of multiple toe nail ONM in 2 elderly patients, who were non-candidates for oral antifungals, by a combination of ciclopirox $8 \%$ nail lacquer and sequential nail peeling with Black peel ${ }^{\circledR}$, a popular anti-acne and cosmetic peel, has been detailed [17]. Black peel is a combination of black acetic acid (BAA), salicylic acid (SA), jasmonic acid (JA), biosulfur, potassium iodide (KI), and lactic acid (LA). The name "Black" stems from its black color, principally imparted by BAA that also gives it a vinegar-like aroma. All major components of this peel combination - BAA, SA, JA, biosulfur, and KI have significant antifungal as well as broad polymicrobial antibiofilm activity. Acetic acid in 5\% concentration or at a concentration to produce a $\mathrm{pH}<3.5$ has been shown to be highly effective in direct killing as well as biofilm matrix penetration of various bacteria as well as Candida spp. [4, 18, 19]. SA as well as aspirin (acetylsalicylic acid) solutions have shown up to $90 \%$ activity against Candida albicans biofilm disruption [20]. A shampoo formulation containing SA $(0.5 \%)$ in combination with zinc pyrithione (2\%) has also been reported to effectively disrupt biofilms formed by Malassezia furfur and $C$. albicans as well as T. rubrum and T. mentagrophytes [21]. Jasmonic acid is a phytohormone with potent activity against pathogenic fungi affecting plants as well as Candida spp. and Aspergillus niger infecting humans [22]. Antifungal properties of sulfur and sulfonated derivatives against human cutaneous mycosis have decent evidence [23]. KI does not have a direct fungistatic, fungicidal, or bactericidal activity. However, it has been suggested that it exerts its cidal effect after getting converted to iodine in vivo by myeloperoxidase, a hydrogen peroxide system of polymorphonuclear cells (present at the site of fungal infection) [24, 25]. KI has also been reported to kill bacterial and fungal cells encased in biofilms extracted from patients with chronic rhinosinusitis [26]. Furthermore, its role as a potentiator of the antibiofilm effect of antimicrobial photodynamic therapy has been reported on multiple occasions $[27,28]$. 
Thus, we now have a new weapon in our arsenal to disrupt the fungal biofilm that poses the biggest challenge to successful treatment of ONM. Akin to repurposing of drugs like ciclopirox [29], the concept of nail peeling for ONM represents repurposing of a physical therapy hitherto indicated for facial lesions like acne, pigmentary disorders, and rejuvenation, for a hardcore clinical dermatologic indication. And in contrast to other antibiofilm approaches, the Black peel offers the apparent advantages of being an economically viable option, having good patient acceptance, practically no adverse effects if the periungual tissues are nicely occluded, and ease of delivery by any dermatologist conversant with the basic principles of chemical peeling.

\section{Disclosure Statement}

The authors have no conflicts of interest to declare. There were no funding sources for this work.

\section{Author Contributions}

Dr. Sidarth Sonthalia developed the concept and was responsible for writing and editing the paper. Dr. Mohamad Goldust assisted in the writing and critical appraisal of the paper.

\section{References}

1 Gupta AK, Carviel J, Shear NH. Antibiofilm Treatment for Onychomycosis and Chronic Fungal Infections. Skin Appendage Disord. 2018 Aug;4(3):136-40.

2 Wu S, Wang Y, Liu N, Dong G, Sheng C. Tackling Fungal Resistance by Biofilm Inhibitors. J Med Chem. 2017 Mar;60(6):2193-211.

3 Finkel JS, Mitchell AP. Genetic control of Candida albicans biofilm development. Nat Rev Microbiol. 2011 Feb;9(2):109-18.

4 Pires RH, da Silva JF, Martins CH, Fusco Almeida AM, Pienna Soares C, Soares MendesGiannini MJ. Effectiveness of disinfectants used in hemodialysis against both Candida orthopsilosis and C. parapsilosis sensu stricto biofilms. Antimicrob Agents Chemother. 2013 May;57(5):2417-21.

5 Wei GX, Xu X, Wu CD. In vitro synergism between berberine and miconazole against planktonic and biofilm Candida cultures. Arch Oral Biol. 2011 Jun;56(6):565-72.

6 Lafleur MD, Sun L, Lister I, Keating J, Nantel A, Long L, et al. Potentiation of azole antifungals by 2-adamantanamine. Antimicrob Agents Chemother. 2013 Aug;57(8):3585-92.

7 Uppuluri P, Nett J, Heitman J, Andes D. Synergistic effect of calcineurin inhibitors and fluconazole against Candida albicans biofilms. Antimicrob Agents Chemother. 2008 Mar;52(3):1127-32.
8 Sharma M, Prasad R. The quorum-sensing molecule farnesol is a modulator of drug efflux mediated by $\mathrm{ABC}$ multidrug transporters and synergizes with drugs in Candida albicans. Antimicrob Agents Chemother. 2011 Oct;55(10):4834-43.

9 De Cremer K, Delattin N, De Brucker K, Peeters A, Kucharíková S, Gerits E, et al. Oral administration of the broad-spectrum antibiofilm compound toremifene inhibits Candida albicans and Staphylococcus aureus biofilm formation in vivo. Antimicrob Agents Chemother. 2014 Dec;58(12):760610.

10 Tan Y, Leonhard M, Moser D, Ma S, Schneider-Stickler B. Antibiofilm efficacy of curcumin in combination with 2 -aminobenzimidazole against single- and mixed-species biofilms of Candida albicans and Staphylococcus aureus. Colloids Surf B Biointerfaces. 2018 Nov; 174:28-34.

11 Stringaro A, Colone M, Angiolella L. Antioxidant, Antifungal, Antibiofilm, and Cytotoxic Activities of Mentha spp. Essential Oils. Medicines (Basel). 2018 Oct;5(4):E112.

12 Feizi S, Taghipour E, Ghadam P, Mohammadi P. Antifungal, antibacterial, antibiofilm and colorimetric sensing of toxic metals activities of eco friendly, economical synthesized $\mathrm{Ag} / \mathrm{AgCl}$ nanoparticles using Malva Sylvestris leaf extracts. Microb Pathog. 2018 Dec; 125:33-42.
13 Chemsa AE, Zellagui A, Öztürk M, Erol E, Ceylan O, Duru ME, et al. Chemical composition, antioxidant, anticholinesterase, antimicrobial and antibiofilm activities of essential oil and methanolic extract of Anthemis stiparum subsp. sabulicola (Pomel) Oberpr. Microb Pathog. 2018 Jun;119:233-40.

14 Farisa Banu S, Rubini D, Shanmugavelan P, Murugan R, Gowrishankar S, Karutha Pandian S, et al. Effects of patchouli and cinnamon essential oils on biofilm and hyphae formation by Candida species. J Mycol Med. 2018 Jun;28(2):332-9.

15 Banga G, Patel K. Glycolic Acid peels for nail rejuvenation. J Cutan Aesthet Surg. 2014 OctDec;7(4):198-201.

16 Daulatabad D, Nanda S, Grover C. Intra-individual Right-left Comparative Study of Medium Depth Peels in Superficial Nail Abnormalities. J Cutan Aesthet Surg. 2017 Jan-Mar; 10(1):28-32.

17 Sonthalia S, Jakhar D, Yadav P, Kaur I. Chemical peeling as an innovative treatment alternative to oral antifungals for onychomycosis in special circumstances. Skin Appendage Disord. 2019. doi: 10.1159/000495152.

18 de Castro RD, Mota AC, de Oliveira Lima E, Batista AU, de Araújo Oliveira J, Cavalcanti AL. Use of alcohol vinegar in the inhibition of Candida spp. and its effect on the physical properties of acrylic resins. BMC Oral Health. 2015 Apr;15(1):52. 
19 Bjarnsholt T, Alhede M, Jensen PØ, Nielsen AK, Johansen HK, Homøe P, et al. Antibiofilm Properties of Acetic Acid. Adv Wound Care (New Rochelle). 2015 Jul;4(7):363-72.

20 Alem MA, Douglas LJ. Effects of aspirin and other nonsteroidal anti-inflammatory drugs on biofilms and planktonic cells of Candida albicans. Antimicrob Agents Chemother. 2004 Jan;48(1):41-7.

21 Dos Santos RM, Dias-Souza MV. Effectiveness of five antidandruff cosmetic formulations against planktonic cells and biofilms of dermatophytes. Saudi J Biol Sci. 2017 Feb;24(2):331-7.

22 Greene SL, Huang YE, Huang LD, Weart IF, Yang SA, Malik S, et al. Antimicrobial compositions comprising a jasmonic acid compound as active agent. European Patent No. EP 1845964B1. Paris: European Patent Office; 2001.
23 Gupta AK, Nicol K. The use of sulfur in dermatology. J Drugs Dermatol. 2004 Jul-Aug; $3(4): 427-31$.

24 Mahajan VK. Sporotrichosis: An Overview and Therapeutic Options. Dermatol Res Pract 2014;2014:272376

25 Grønseth T, Vestby LK, Nesse LL, Thoen E, Habimana $\mathrm{O}$, von Unge $\mathrm{M}$, et al. Lugol's solution eradicates Staphylococcus aureus biofilm in vitro. Int J Pediatr Otorhinolaryngol. 2017 Dec;103:58-64.

26 Masood R, Wrobel B, Dale H. A comparison of the effectiveness of tincture of iodine and potassium iodide on chronic sinusitis patients with biofilm. J Neurol Neurosurg. 2017;6(3): 555690 .
27 Wen X, Zhang X, Szewczyk G, El-Hussein A, Huang YY, Sarna T, et al. Potassium Iodide Potentiates Antimicrobial Photodynamic Inactivation Mediated by Rose Bengal in In Vitro and In Vivo Studies. Antimicrob Agents Chemother. 2017 Jun;61(7):e00467-17.

28 Vecchio D, Gupta A, Huang L, Landi G, Avci $\mathrm{P}$, Rodas $\mathrm{A}$, et al. Bacterial photodynamic inactivation mediated by methylene blue and red light is enhanced by synergistic effect of potassium iodide. Antimicrob Agents Chemother. 2015 Sep;59(9):5203-12.

29 Rangel-Vega A, Bernstein LR, MandujanoTinoco EA, García-Contreras SJ, GarcíaContreras R. Drug repurposing as an alternative for the treatment of recalcitrant bacteria infections. Front Microbiol. 2015 Apr;6:282. 